\title{
Capacitive gas-phase detection in liquid nitrogen
}

\author{
Christoph Kandlbinder ${ }^{1}$, Alice Fischerauer ${ }^{1}$, Mario Mösch ${ }^{1}$, Tobias Helling ${ }^{1}$, Gerhard Fischerauer ${ }^{1}$, \\ and Martin Siegl ${ }^{2}$ \\ ${ }^{1}$ Chair of Measurement and Control Systems, Center of Energy Technology (ZET), University of Bayreuth, \\ 95440 Bayreuth, Germany \\ ${ }^{2}$ Institute of Space Systems, German Aerospace Center (DLR), 28359 Bremen, Germany
}

Correspondence to: Chistoph Kandlbinder (mrt@uni-bayreuth.de)

Received: 13 August 2016 - Revised: 1 February 2017 - Accepted: 12 February 2017 - Published: 2 March 2017

\begin{abstract}
The main and upper stages of heavy lift launchers for space applications are often fuelled by cryogenic liquids. In order to enable the re-ignition of a cryogenic upper stage for orbital changes, it is crucial to study the behaviour of these fluids in microgravity. As gaseous bubbles entering the fuel lines of the engine can cause the destruction of the engine, these bubbles are a risk for the functionality of the re-ignition mode. To measure an evolving gaseous phase and its volume, a capacitive measurement system for two-phase mixtures was realised. Its electrodes are arranged in such a way that phase changes inside a vessel can be detected without parasitic heating under cryogenic conditions. Two cases have been investigated: a fill-level measurement involving a large gas bubble above a homogenous liquid on the one hand, and the identification of a bubble stream inside a liquid on the other hand. The system concept was tested in a cryogenic environment allowing the controlled generation of bubble streams inside liquid nitrogen and of a contiguous gaseous volume above the liquid. The characteristics of the measurable capacitances of different pairs of electrodes were experimentally determined and compared with finite-element simulations (Ansys). In addition, the electrical flux density was computed to corroborate the simulated capacitance curves with theoretical statements. The experimental findings closely agree with the simulated results if possible disturbances due to the characteristics of the capacitance measurement hardware are properly taken into account. Thus, by measuring various capacitances, it was possible to determine the level up to which a receptacle inside a liquid-nitrogen tank was filled with liquid (the space above the liquid being taken up by gaseous nitrogen), to identify the existence of a bubble stream in the liquid nitrogen and to demonstrate that the capacitance measurement results enable one to differentiate between the two cases.
\end{abstract}

1

For future space vehicles like the Ariane 6 rocket of the European Space Agency (ESA), re-ignition of the upper stage driven by cryogenic liquids will play a key role. Upper stages already surrounding Earth must re-ignite their thrust engine to change into a higher orbit. This possibility could open up new opportunities for space applications, like releasing one part of the payload into a lower orbit and another part into a higher orbit (Stolten and Emonts, 2016).

In order to observe fluid phenomena occurring in microgravity and to eventually influence their behaviour, it is crucial, especially for partially filled tanks, to determine the location of the liquid phase and the topology of the free surface.
As capillary forces are dominant under microgravity conditions, these forces determine the shape and position of the free surface. Additionally, gas bubbles can come into existence by sloshing, changes in gravitational forces, or external heat input. If these bubbles enter the fuel lines of the turbopumps, the pumps can be destroyed by cavitation effects. This would render a re-ignition impossible. To analyse these phenomena, experiments with suitable measurement systems are inevitable (Stolten and Emonts, 2016; Siegl et al., 2015).

Such measurement systems should only generate a negligible amount of heat input into the cryogenic liquid. In addition, they are required to work under cryogenic conditions and to determine the spatial distribution of gaseous phases (bubbles) in a non-invasive manner. 


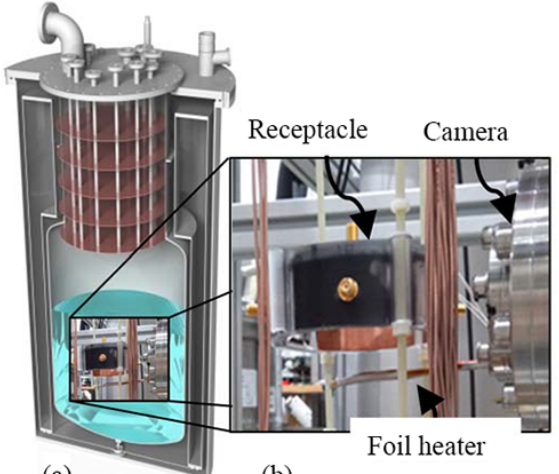

(a)

(b)

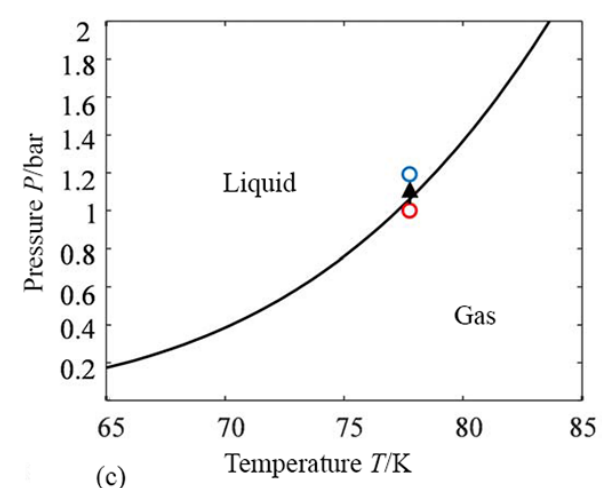

Figure 1. Cryostat as measurement environment. (a) Structure of the cryostat. (b) Built-in cylindrical receptacle with foil heater and camera. (c) Pressure-temperature $(p-T)$ diagram of liquid nitrogen $\left(\mathrm{LN}_{2}\right)$ (Span et al., 2000).

Current measurement systems for cryogenic environments use gamma-ray-based (Carapelle and Colette, 2005), microwave-based (Ohira, 2004), radio-frequency-based (Filippov et al., 2013), resistive, or capacitive (Jiang and Zhang, 2011) principles. Some of them intrude into the volume under investigation, and some can lead to a local heat input or other parasitic effects (Mukhopadhyay, 2010). Among them, capacitive sensors promise a high potential to not only determine the position of the surface of a liquid phase (i.e. the position of the liquid-to-gas interface), but to also be costefficient, simple, and to show fast reaction times (Carapelle and Colette, 2005; Medeova et al., 1998; De Kerpel et al., 2013). They neither disturb the liquid movement nor do they heat the liquid. The required electrodes can be integrated into walls of fuel lines or smaller tanks inside space systems in such a manner that the tank geometry need not be modified (Nurge et al., 2003). The capacitive observability of a single gas-liquid phase boundary under cryogenic conditions has been demonstrated by Hennes and Barnard (1964) and Kügler et al. (1984) with liquid nitrogen $\left(\mathrm{LN}_{2}\right)$ as the liquid; based on this, a fill-level resolution of $\pm 1 \mathrm{~mm}$ was reached. Successful fill-level measurements have also been reported by Matsumoto et al. (2011) with liquid hydrogen $\left(\mathrm{LH}_{2}\right)$. The earlier studies, of course, did not have access to modern electronics and thus had to limit themselves to very specific geometries and measurement purposes. But even the recent work in Matsumoto et al. (2011) did not aim for a detailed investigation of the electrode geometry, its influence on the measurable capacitances, the information which may be extracted from these capacitances on quantities other than the fill level, and the possibly error-producing influence of the capacitance measurement hardware. We have designed and tested a capacitive system for gas-phase detection under cryogenic applications. We specifically investigated the capacitance measurement process itself and how well the system can differentiate between single-phase boundaries (large gas bubble above a sea of $\mathrm{LN}_{2}$ as it is assumed in filllevel measurement) and multiple phase boundaries (stream of many small gas bubbles rising in a sea of $\mathrm{LN}_{2}$ ) as such effects are likely to occur in zero-gravity environments.

\section{Hardware}

\subsection{Cryostat}

Since the measurement system that will be described later has to be tested in cryogenic liquids, a cryostat is used as a test environment (Fig. 1a and b). The inner tank of the cryostat could be pressurised up to 4 bar while the space between the inner and outer tank could be filled with $\mathrm{LN}_{2}$ for further thermal isolation from the environment. Inside the inner tank, a foil heater was mounted for defined bubble generation by local heat input into the $\mathrm{LN}_{2}$.

The liquid-vapour system in the cryostat was either kept on the boiling curve or in a slightly subcooled state. By adjusting the pressure, it was then possible to control the volume of gaseous nitrogen inside the tank. When the pressure rose inside the cryostat upon closure of a valve, the gaseous volume shrank because of condensation processes, and the liquid assumed a subcooled state. This is visible in a pressure-temperature ( $p-T$-) diagram (Fig. 1c). The working point is marked by a red circle located on or slightly below the boiling curve. When the pressure rises, the working point moves upward into the region associated with the liquid state (blue circle). As a consequence, a gaseous volume trapped in a liquid phase condensates. By using these principles, the gas-to-liquid ratio inside this trapped volume can be controlled.

However, the foil heater can generate bubbles by locally heating the $\mathrm{LN}_{2}$ so that subcooled nucleate or film boiling can come into existence. These generated bubbles can rise until they reach the surface of $\mathrm{LN}_{2}$. If they are trapped while rising onto the surface by special equipment, they coalesce and form a gaseous volume inside the $\mathrm{LN}_{2}$. 


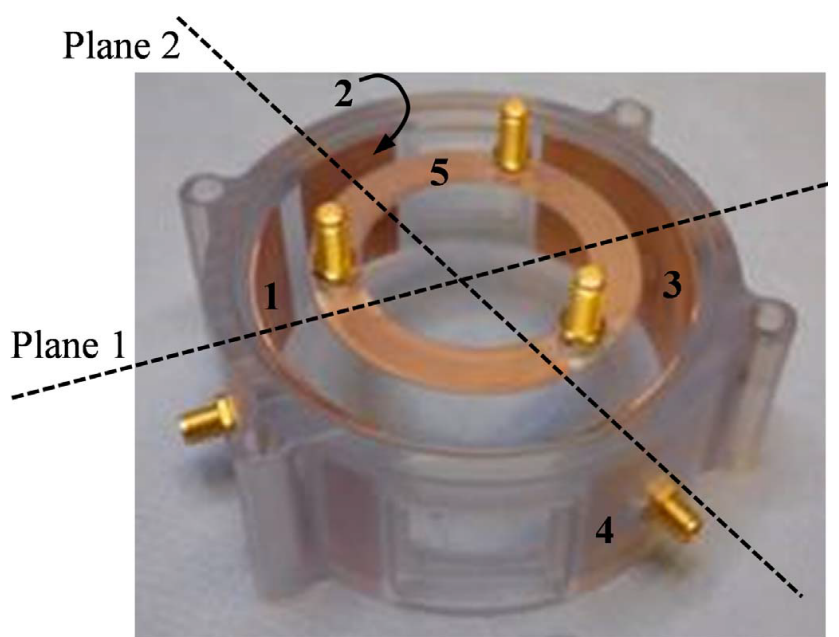

Figure 2. Photograph of the circular cylindrical polycarbonate receptacle with five electrodes of different shapes.

\subsection{Measurement system}

The exemplary configuration used in this work consists of a circular cylindrical polycarbonate receptacle open at the bottom, with a lid on top and mounted inside a $\mathrm{LN}_{2}$-filled cryostat. This configuration allows the regulation of the fill level by (de-) pressurisation, as is the case in many space systems. Bubbles rising from the liquid volume below the receptacle are trapped by it. These local phase changes from liquid to gas are recognised by measuring the capacitances between different electrodes located on the inner surface of the cylinder wall and the lid. The basis of the liquid-gas differentiation is the fact that the permittivities of liquid and gaseous nitrogen differ from each other $\left(\varepsilon_{\mathrm{r}, \mathrm{LN}_{2}} \approx 1,43, \varepsilon_{\mathrm{r}, \mathrm{N}_{2}(\mathrm{~g})} \approx 1,0\right)$ (Murphy and Morgan, 1937).

The geometry of the cylinder and its electrodes is shown in Fig. 2. The receptacle has an inner diameter of $80 \mathrm{~mm}$ and a height of $30 \mathrm{~mm}$. Electrodes 2 and 4 have areas of $900 \mathrm{~mm}^{2}$ and electrodes 1 and 3 have areas of $1800 \mathrm{~mm}^{2}$. The circular ring electrode at the top of the lid has inner and outer diameters of 40 and $60 \mathrm{~mm}$, respectively. The closest distance between electrodes 2 and 5 is $10 \mathrm{~mm}$, and that between electrodes 1 and 3 is $80 \mathrm{~mm}$. The geometry was chosen so as to evaluate the differences in capacitance due to different electrode geometries and their arrangement on the one hand, and to make an optical inspection possible on the other hand. The dashed lines shown in Fig. 2 mark the intersections of vertical planes (planes containing the cylinder axis) and the top side of the receptacle lid; field simulations in these planes will later be presented to support certain statements about the characteristics of the measurement system.

The desired capacitances were measured with integrated circuits (ICs) of the type Acam PCap02AD. This IC calculates a capacitance by evaluating the discharge time of the unknown capacitor in series with a known resistor relative

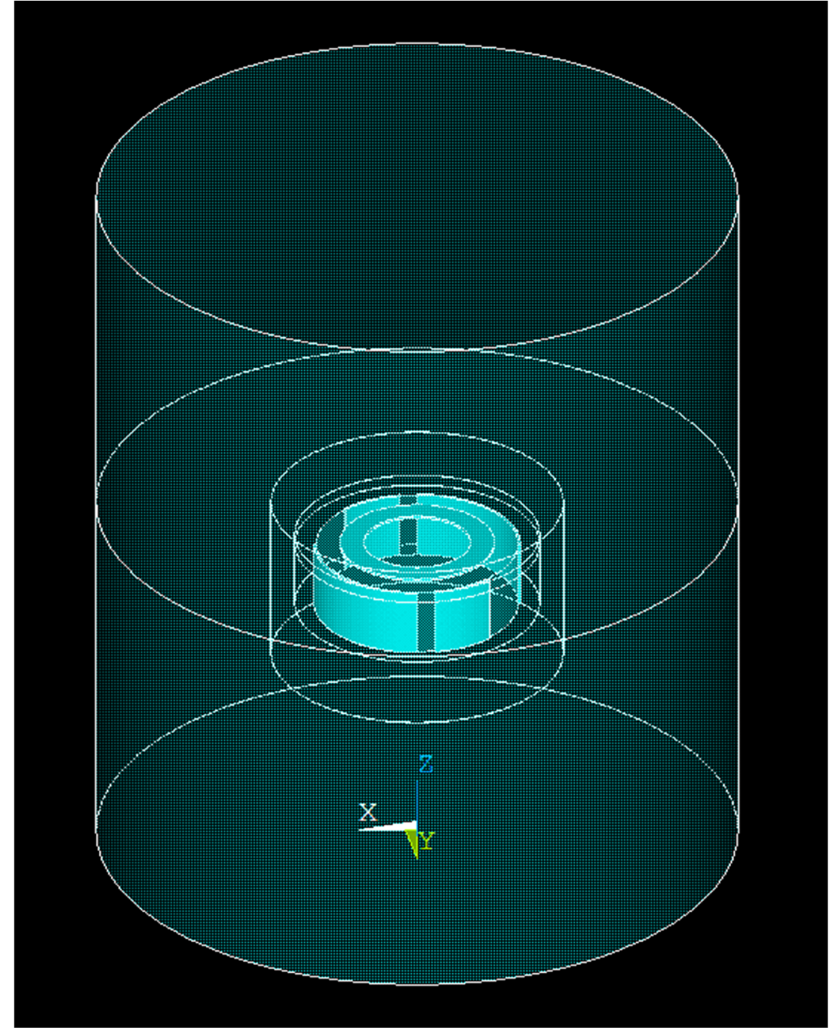

Figure 3. FE geometry of the receptacle and its environment.

to the discharge time of a known capacitor-resistor combination. The IC handles both ground capacitances (one electrode active, all other electrodes of a multi-electrode system grounded) and mutual capacitances (two electrodes active, all other electrodes grounded) (Acam messelectronic $\mathrm{GmbH}$, 2014; Schlegl et al., 2013). The respective advantages of these two measurement modes in our application have been compared and will be discussed after simulation results for the capacitances have been presented (see end of Sect. 3).

In the experiments conducted, gas bubbles were generated in $\mathrm{LN}_{2}$ in a defined way by using the foil heater as described before. These bubbles could be accumulated under the lid inside the cylindrical receptacle and united to form an expanding gaseous phase. By using a camera that observes the cylinder from the side, the fractional gas-phase ratio inside the cylinder could be observed while the capacitances were measured simultaneously.

\section{Modelling and simulation of capacitances}

For validation and interpretation purposes, the measurements were accompanied by finite-element (FE) simulations (Ansys). Figure 3 shows the FE geometry model. Some care has been exercised to reproduce the real geometry of Fig. 1 well and to use a dense mesh close to the fine structures of the receptacle. 

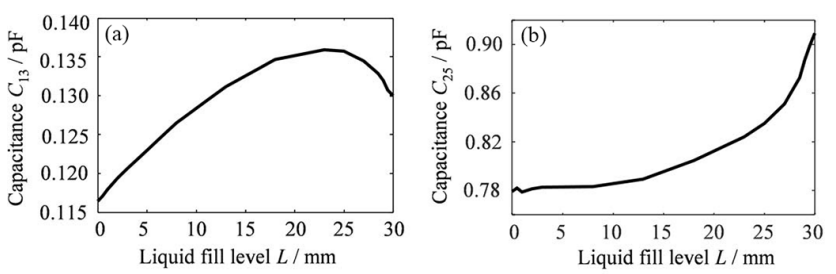

Figure 4. Simulated mutual capacitances of different pairs of electrodes as a function of the $\mathrm{LN}_{2}$ fill level. (a) $C_{13}$ (b) $C_{25}$.
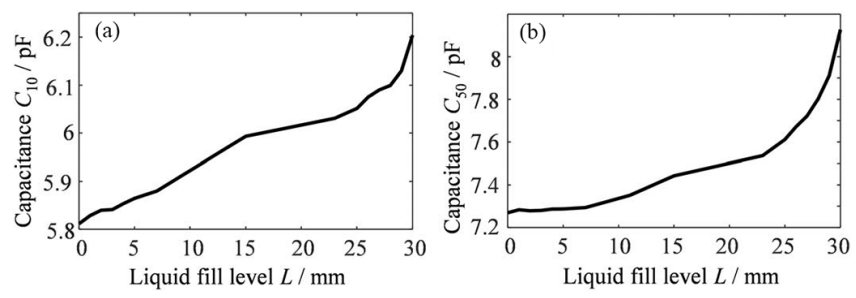

Figure 5. Simulated ground capacitances of different electrodes as a function of the $\mathrm{LN}_{2}$ fill level. (a) $C_{10}$ (b) $C_{50}$.

Bubbles rising in the $\mathrm{LN}_{2}$ coalesce inside the receptacle, and as a consequence the liquid fill level $L$ inside the receptacle drops. The results of the simulation in Fig. 4 demonstrate how selected mutual capacitances $C_{i j}$ (measured between electrodes $i$ and $j$ ) depend on $L$. The numbering of the electrodes is the same as in Fig. 2.

A look at Fig. 4 a reveals that the capacitance $C_{13}(L)$ depends quasi-linearly on $L$ at small fill levels $(L<20 \mathrm{~mm})$. At higher fill levels, $C_{13}$ first peaks at $L \approx 23 \mathrm{~mm}$ and then drops again for fill levels between 23 and $30 \mathrm{~mm}$. For reasons of sensitivity and uniqueness, $C_{13}$ only lends itself for the measurement of fill levels below $20 \mathrm{~mm}$. In contrast, with $C_{25}(L)$, the slope and therefore the sensitivity of the characteristic curve is nearly complementary (in other words, the functional graph $C_{13}(L)$ is concave from below whereas the graph $C_{25}(L)$ is convex from below; Fig. 4b). This mutual capacitance hardly changes with $L$ at small fill levels ( $L<20 \mathrm{~mm}$ ), but is strongly sensitive to $L$ at higher levels ( $L=20 \ldots 30 \mathrm{~mm}$ ). This makes sense as electrode 5 is located on the lid, and is therefore more affected by the fill level the higher the level is.

Similar, but not completely identical, remarks apply to ground capacitances such as $C_{10}(L)$ and $C_{50}(L)$ (Fig. 5). Their sensitivities (rates of change) increase with $L$ at high fill levels, especially when the receptacle is nearly full of $\mathrm{LN}_{2}$. The overall change in capacitance from $L=0$ to $L=L_{\max }=30 \mathrm{~mm}$ is higher for ground capacitances than for mutual capacitances by an order of magnitude (some $0.1 \mathrm{pF}$ vs. some $0.01 \mathrm{pF}$ ). Obviously, ground capacitances should be measured to observe phase changes near the electrodes involved. And mutual capacitances are needed for electrical capacitance tomography (ECT) to identify phase interfaces in the volume between the electrodes (Mühlbacher-Karrer

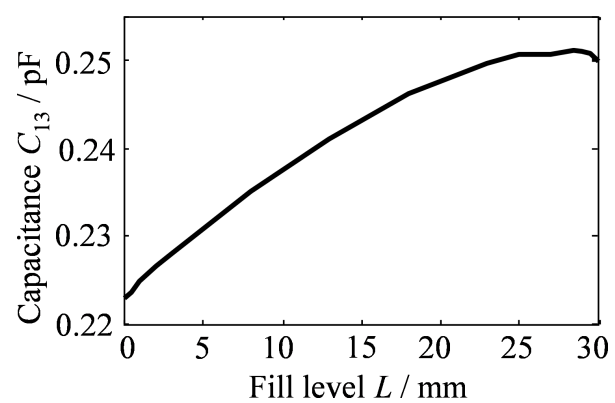

Figure 6. Simulated mutual capacitance $C_{13}$ for a ground potential deviation of $2 \%$ of the active-electrode potential.

and Zangl, 2015; Wang et al., 2010). The measurement of these mutual capacitances is influenced by non-ideal ground connections. This can be seen if Fig. 4 a $\left(C_{13}\right.$ with perfectly grounded electrodes 2,4 , and 5$)$ is compared to Fig. $6\left(C_{13}\right.$ with electrodes 2,4 , and 5 on a potential that is $2 \%$ of the active-electrode potential). Hence, when interpreting measured capacitances, one must take such common imperfections into account.

\section{Electric-field simulation}

For a better understanding of the simulated capacitances, the electrostatic field in the region close to the electrode-carrying receptacle was investigated by FE calculations. As an example, this will first be discussed by looking at the potential distribution associated with the measurement of the mutual capacitance $C_{13}(L)$. The dashed lines in Fig. 2 indicate the lines of intersection between the plane of projection and the two planes, normal to the plane of projection, in which the electric flux density $\boldsymbol{D}(\boldsymbol{r})$ has been computed.

Figure 7 shows the magnitude and the field lines of $\boldsymbol{D}(\boldsymbol{r})$ for three different $\mathrm{LN}_{2}$ fill levels in plane 1 (which runs through the centres of electrodes 1 and 3) when electrode 3 is excited. Without gas inside the receptacle (Fig. 7a), the electric field in the region $\mathrm{G}$ between electrodes 1 and 5 (marked by a black circle in Fig. 7a) is weaker than near the lower part of electrode 1 (note the colour codes). The field lines starting on the active electrode 3 end in part on electrode 1 and in part on electrode 5. With increasing gas volume (corresponding to decreasing $\mathrm{LN}_{2}$ level), the flux density in region $\mathrm{G}$ increases (Fig. 7b). Fewer field lines than before end on electrode 5 , because this electrode borders on the gaseous phase and the field concentrates in the $\mathrm{LN}_{2}$ with its higher permittivity. As the major part of electrode 1 reaches into the $\mathrm{LN}_{2}$, some field lines that ended on electrode 5 in Fig. 7a now end on electrode 1, and the capacitance $C_{13}$ now is greater than in Fig. 7a. At even larger gas volumes (lower $\mathrm{LN}_{2}$ levels), the field in region $\mathrm{G}$ gets weaker again (Fig. 7c). Now the major part of electrode 1 is in contact with gaseous nitrogen, and the 
(a)

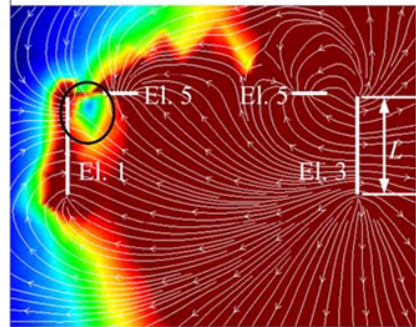

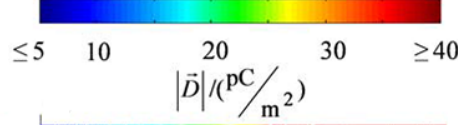

(b)

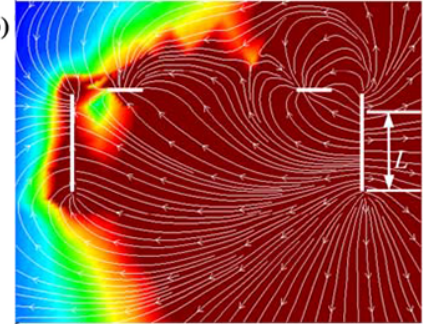

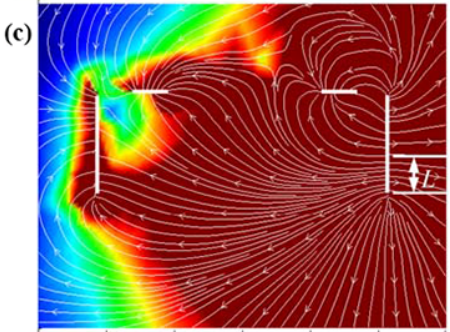

Figure 7. Magnitude (colour-coded) and field lines of the electric flux density in plane 1 (see Fig. 2) when electrode 3 is active. The three figures correspond to three different $\mathrm{LN}_{2}$ fill levels. (a) $L=30 \mathrm{~mm}$ (cylinder fully filled with $\mathrm{LN}_{2}$ ). (b) $L=25 \mathrm{~mm}$. (c) $L=10 \mathrm{~mm}$. The black circle in (a) marks the region of interest $\mathrm{G}$, in which the electric flux density is further examined, as described in the text.
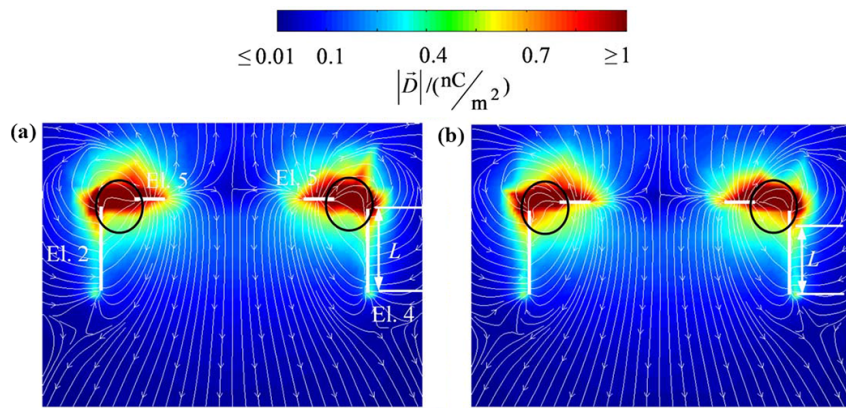

Figure 8. Simulated magnitude (colour-coded) and field lines of the electric flux density in plane 2 (see Fig. 2) when electrode 5 is active. The two figures correspond to two different $\mathrm{LN}_{2}$ fill levels. (a) $L=30 \mathrm{~mm}$ (cylinder fully filled with $\mathrm{LN}_{2}$ ). (b) $L=25 \mathrm{~mm}$. The black circles mark two regions that are further considered for analysis, as described in the text.

electrode has lost its advantage at "catching" the field lines. As a consequence, $C_{13}$ decreases with decreasing fill level.

Another example is shown in Fig. 8. It refers to plane 2 of Fig. 2 (which runs through the small electrodes 2 and 4), and the active electrode is electrode 5. Without gas in the receptacle, the electric flux density is highest in the corner regions $\mathrm{G}$ (marked by black circles) between the top electrode 5 and the sidewall electrodes 2 and 4 (Fig. 8a). The situation is similar at lower $\mathrm{LN}_{2}$ levels (Fig. $8 \mathrm{~b}$ ), but the field is somewhat weaker in $\mathrm{G}$. This explains the drop in capacitance $C_{25}$ : there are just fewer field lines running from electrode 2 through the gaseous phase to electrode 5 than before, when the volume was completely filled with $\mathrm{LN}_{2}$.

Figure 9 is an extension of Fig. 8 to even lower fill levels (half-full receptacle in the case of Fig. 9a and completely empty receptacle in the case of Fig. 9b). The maximum flux density is smaller than before $\left(0.5\right.$ vs. $\left.1 \mathrm{nC} \mathrm{m}^{-2}\right)$, and the field is more evenly distributed inside the gaseous region than before. Also, the field lines are almost straight inside the gaseous volume and somewhat chaotic outside, which
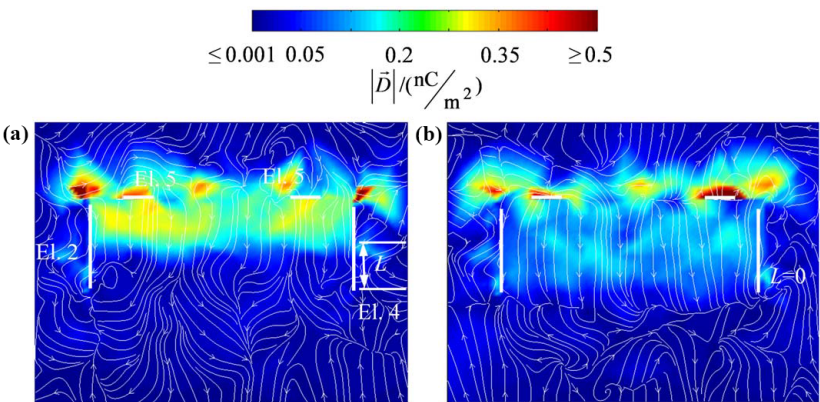

Figure 9. Simulated magnitude (colour-coded) and field lines of the electric flux density in plane 2 (see Fig. 2) when electrode 5 is active. The two figures correspond to two different $\mathrm{LN}_{2}$ fill levels. (a) $L=15 \mathrm{~mm}$ (cylinder half filled with $\mathrm{LN}_{2}$ ). (b) $L=0 \mathrm{~mm}$ (no $\mathrm{LN}_{2}$ present in the cylinder).

also shows that the field strength varies little from the top of the receptacle to the bottom and has its highest change at the top. The capacitance only changes very little compared to the changes observed when the gaseous phase begins to emerge. Note that the local density of the field lines in the figures does not represent the local field strength (as it should, but the software used simply selects a few lines regardless of the local field strength).

To visualize the variations in the region of the (left) black circle, the mean value of the magnitude of the electrical flux density in this region is shown in Fig. 10 for changing $\mathrm{LN}_{2}$ fill levels. The results bear some resemblance to Fig. 4, in which the change of capacitance over the fill level is plotted. Hence, the capacitance curves can be explained by the change of the flux density in the region near the two electrodes making up the measured capacitor.

\section{Measurements and results}

Different receptacle-filling and receptacle-emptying cycles were studied experimentally. At rising $\mathrm{LN}_{2}$ fill levels, se- 

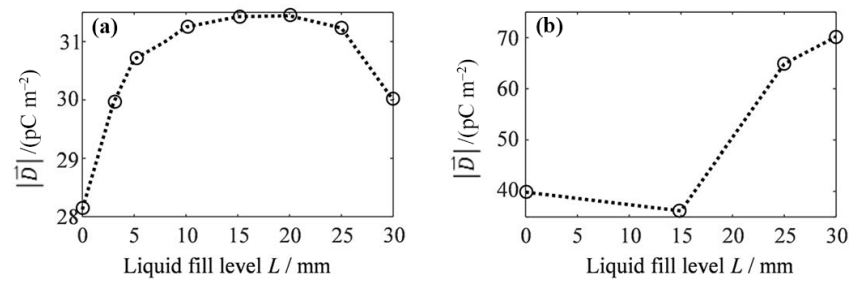

Figure 10. Simulated electric flux density magnitude for different fill levels in (a) plane 1, when electrode 3 is active (see Fig. 7); (b) plane 2, when electrode 5 is active (see Figs. 8 and 9). The circles mark the simulated data points, the dotted lines only serve to guide the eye.

lected capacitances were measured and the gas-liquid interface, as well as the activity of bubbles, was optically observed by the camera. The actual fill level was determined by using the pictures from the camera. As the resolution of the camera and the light source in the cryostat did not suffice for precise measurements, the fill level can only be determined with an uncertainty of $\pm 2 \mathrm{~mm}$ from the video camera images. Figure 11 shows the measured $\mathrm{LN}_{2}$ fill level $L$ as a function of time for a typical experiment in which the mutual capacitances $C_{13}$ and $C_{25}$ were measured. The fill level $L$ was calculated by inverting the theoretical characteristic curves $C_{13}(L)$ and $C_{25}(L)$ from the results of the simulations (Fig. 6a, b). Also, the dashed curve with the associated light "belt" shows the fill level determined from the video camera images with an uncertainty band of $\pm 2 \mathrm{~mm}$.

At the beginning of the measurement $(t=0 \mathrm{~s})$, the receptacle was partially filled with $\mathrm{LN}_{2}$. After a decrease of the pressure in the cryostat, the existing gaseous phase inside the receptacle expanded until it was eventually completely filled with gaseous nitrogen $(L=0)$ at $t \approx 150 \mathrm{~s}$. After that point, the pressure was increased again, and the gas-liquid phase boundary rose almost linearly with time until the receptacle was completely filled with $\mathrm{LN}_{2}(L=30 \mathrm{~mm})$ at $t \approx 450 \mathrm{~s}$. The heater was turned on at $t \approx 650 \mathrm{~s}$. After that, bubbles rose in the $\mathrm{LN}_{2}$ reservoir below the receptacle and replaced $\mathrm{LN}_{2}$ in the receptacle. After the beginning of this replacement process, the $\mathrm{LN}_{2}$ fill level decreased with time. As expected, $C_{13}$ is sensitive to the beginning of the bubble stream $(t \approx 650 \mathrm{~s})$ as the presence of bubbles leads to a lower effective permittivity of the fluid between electrodes 1 and 3 . If one neglects this effective-permittivity effect and merely assumes a planar interface between a gaseous phase above a liquid "sea", one underestimates the $\mathrm{LN}_{2}$ fill level. In contrast to this, $C_{25}$ is not influenced by the bubble stream. There is only a small amount of field lines from electrode 5 to electrode 2 running through the bubble stream, as can be seen from the geometry of the cylinder and the position of the heater underneath. As mentioned before, $C_{13}(L)$ should be used to estimate the $\mathrm{LN}_{2}$ fill level $L$ in the region from 0 to $20 \mathrm{~mm}$ whereas $C_{25}(L)$ is more suitable for $L$ between 15 and $30 \mathrm{~mm}$. As shown by
Fig. 11, the noise in the estimated $L$ increases dramatically if $L$ is extracted from the "wrong" capacitance. The two capacitively measured curves agree quite well with the visually observed fill level, unless a bubble stream disturbs the measurement (in which case the capacitive measurement can and must be corrected by taking into account the results from several capacitances) and there are low fill levels (in which case the differences are attributed to reading errors in the interpretation of the video camera images - low fill levels were hard to make out on the images, and the uncertainty is likely to exceed $2 \mathrm{~mm}$ in these cases).

We would like to draw attention to the fourth photograph of the receptacle from the left in Fig. 11, which is a freezeframe image of the video at $t \approx 650 \mathrm{~s}$. At this instant, the $\mathrm{LN}_{2}$ fill level $L$ was decreasing owing to the bubble stream. Because of the bubbles, the surface of the liquid is very agitated: it "boils".

The entire situation can be modelled by a cylinder with the permittivity of $\mathrm{LN}_{2}$ (= receptacle full of $\mathrm{LN}_{2}$ ) in which there is embedded a slender cylinder of lower permittivity (bubble stream between electrodes 1 and 3 ). An effective relative permittivity of $\varepsilon_{\mathrm{r}}=1.1$ was chosen for the bubble stream region, to represent a high bubble content. The bubble-stream region in the simulation was chosen such that it coincided with the bubble-stream region visible in the camera-recorded video. The simulation results are summarized in Fig. 12, where the numbering of the electrodes and the notation for the crosssectional planes is the same as in Fig. 2. It is obvious that, for example, the mutual capacitance $C_{13}$ and the ground capacitance $C_{30}$ will respond to the presence of the bubble stream as the associated field lines "probe" (run through) the bubblestream region (Fig. 12a, b). In contrast, capacitance $C_{25}$ will be hardly affected by the presence of the bubble stream as the associated field lines do not run through the bubble-stream region (Fig. 12c, d). Hence, not only can the fill level in a defined volume be detected, but so too can a change in permittivity (like a bubble stream or bubbles) inside a confined volume.

As a further corroboration of the calculation and measurement results, the gas volume increase due to a depressurization of the cryostat was observed. The change of the fill level from full to empty was nearly linear with time, so that the simulated capacitance curves from Fig. 6 can be superimposed on the measured capacitance curves. For the mutual capacitances as well as for the grounded capacitances, the simulation results and the experimental findings agree very well (Fig. 13). The following conclusions can be drawn from these results:

- The measurement results can be correctly interpreted in a quantitative as well as in a qualitative way by using numerical simulations (of capacitances).

- Ground-potential errors occur in practical capacitance measurements. These are not negligible and have to be taken into account for the interpretation of measured 


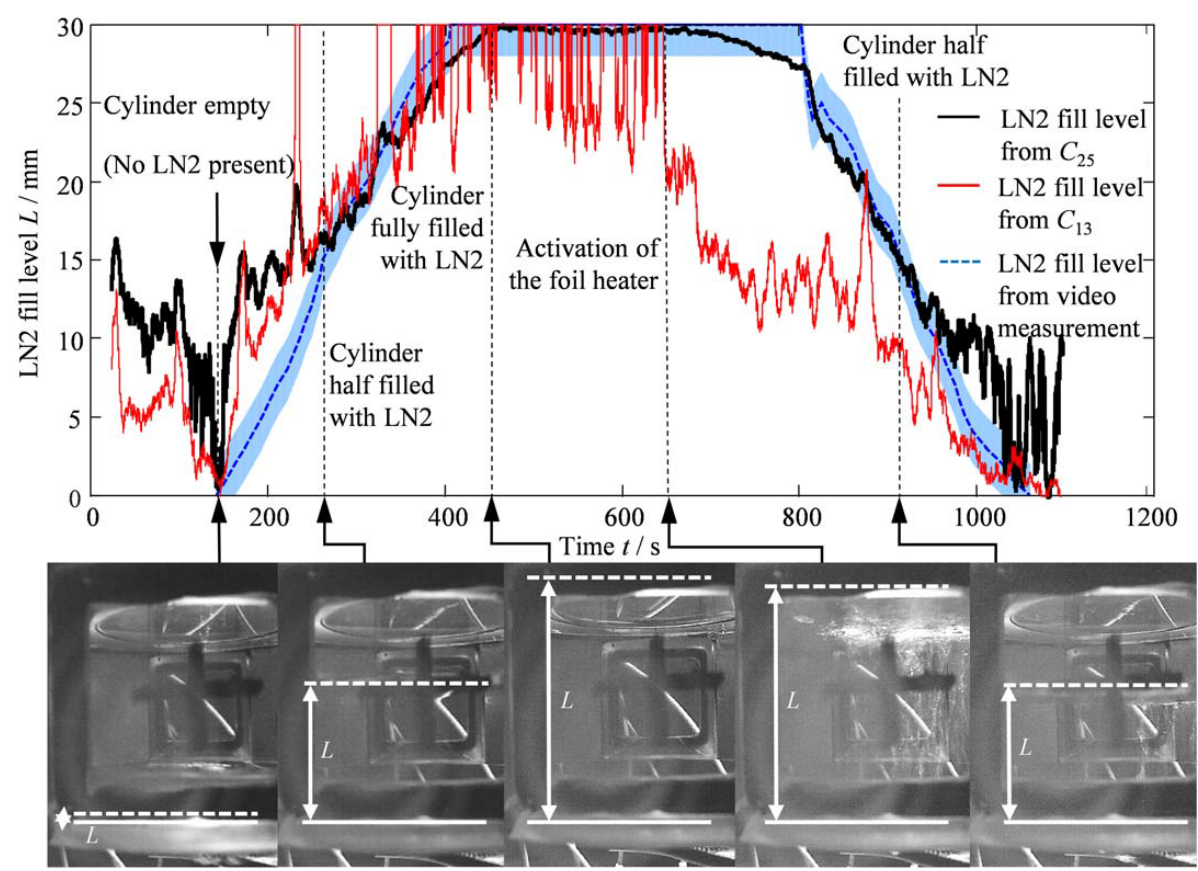

Figure 11. $\mathrm{LN}_{2}$ fill level inside the receptacle measured via electrode pair 1-3 (red curve) and via electrode pair 2-5 (black curve) compared to the visually determined fill level (video camera images; dashed line with an uncertainty band of $\pm 2 \mathrm{~mm}$ ). The photographs at the bottom are freeze-frame images from the video camera and serve to visualize selected situations.

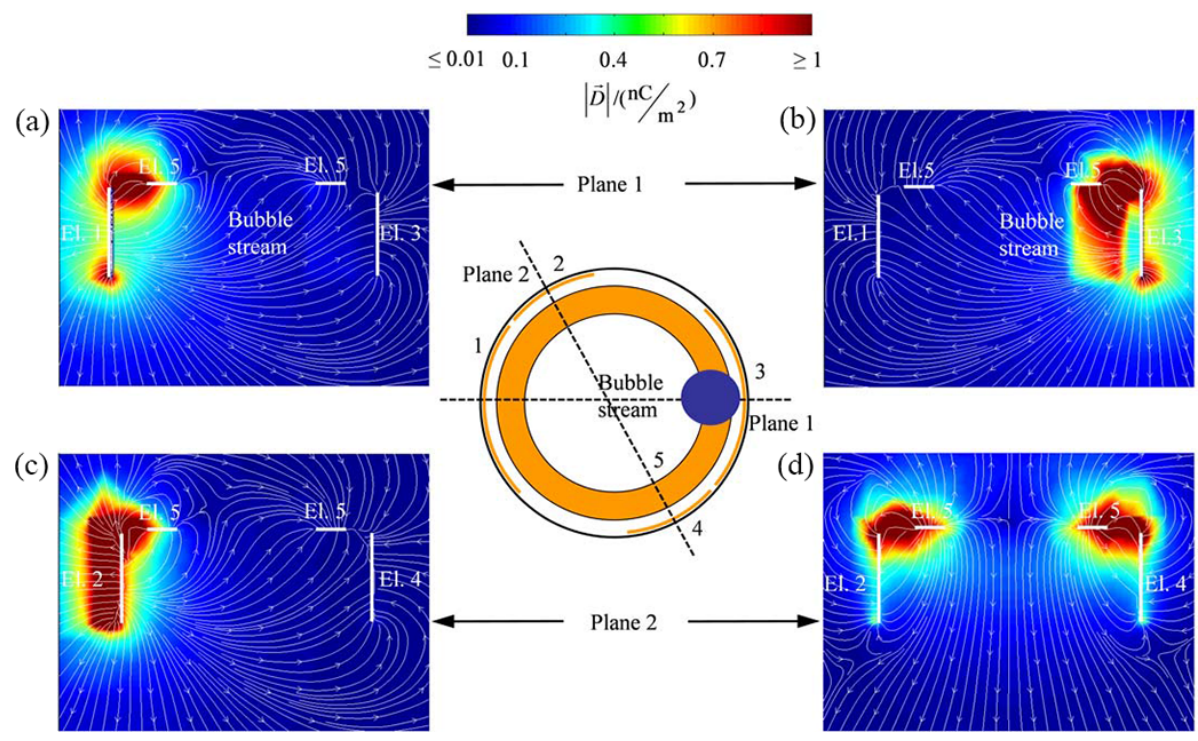

Figure 12. Simulated magnitude (colour-coded) and field lines of the electric flux density in the presence of a bubble stream at the position indicated in the center sketch. (a, b) Field in plane 1 with excitation of (a) electrode 1 and (b) electrode 3. (c, d) Field in plane 2 with excitation of (c) electrode 2 and (d) electrode 5.

data. This can be done by using a model-based approach.

- The measured capacitances are noisy. The amount of noise depends on both the capacitance and the permittivity distribution ( $\mathrm{LN}_{2}$ fill level). In general, the curves have to be smoothed. In the case of Fig. 13a, a moving average filter with a window size of 200 was used. The measurement frequency was $25 \mathrm{~Hz}$, so that 200 measured points represent a time of $8 \mathrm{~s}$. With the ground capacitances (larger than the mutual capacitances), the curves may not need to be smoothed. In the case of Fig. 13b, no smoothing filter was used. 

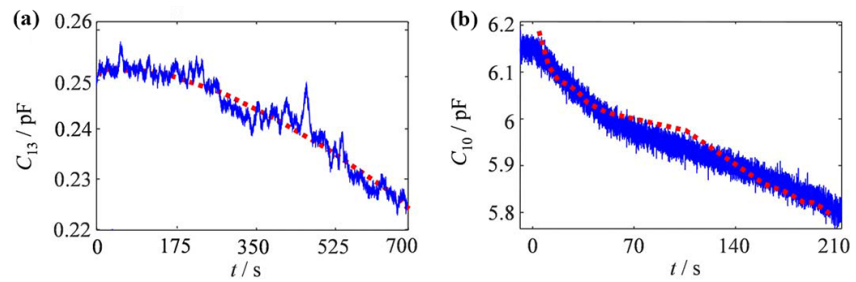

Figure 13. Measured capacitances (solid blue lines) and finiteelement simulations (dotted red lines) for a dynamic experiment with time-varying gas volume inside the receptacle. Both curves refer to a time interval in which the gas volume increases (decrease of the $\mathrm{LN}_{2}$ level). (a) Mutual capacitance $C_{13}$. The same ground-potential error as in Fig. 6 has been assumed in the simulation. Smoothing of the measured curve as described in the text. (b) Ground capacitance $C_{10}$.

- The measured capacitances contain an additive offset that is characteristic for every combination of electrodes. The offset is due to cable capacitances and has to be subtracted from the raw data.

- Our specific hardware implementation could handle capacitance changes of some femtofarads without problems.

- The system can distinguish between a change in fill level and bubbles or another source of change in permittivity occurring inside a confined volume by using the information supplied by multi-electrode arrangements.

\section{Conclusions}

It has been demonstrated that phase changes in $\mathrm{LN}_{2}$ can be recognized in situ by measuring the electrical capacitance between different electrodes of a multi-electrode system. The method is minimally invasive as it does not introduce heat into the $\mathrm{LN}_{2}$ or otherwise disturb the fluid.

This may be generalized to infer that, depending on the geometry of the electrodes, phase changes of fluids with comparatively low changes in relative permittivity can be detected using measured mutual and ground capacitances. The properties of the system can be well described by using dedicated finite-element simulations. Both the agreement between simulation and electrical measurements and the agreement between simulation and optical (camera-based) measurements are very good.

Data availability. Original data of the publication: doi:10.15495/M-10150282-0001 (Kandlbinder et al., 2017). The data was used to produce Figs. 1, 4, 5, 6, 7, 8, 9, 10, 11, 12, and 13 .
Competing interests. The authors declare that they have no conflict of interest.

Acknowledgements. Parts of the presented work were carried out under contract from the European Space Agency (ESA), the support of which is gratefully acknowledged.

Edited by: A. Lloyd Spetz

Reviewed by: two anonymous referees

\section{References}

Acam messelectronic GmbH: Data Sheet "PCap02A - Single-chip Solution for Capacitance Measurement", Vol. 1, vers. 1.6, 29 May 2014.

Carapelle, A. and Colette, J.: Gamma-ray attenuation for measuring cryogenic slush mixture density, Nucl. Instr. Meth. Phys. Res. B, 229, 111-116, 2005.

De Kerpel, K., Ameel, B., T'Joen, C., Canière, H., and De Paepe, M.: Flow regime based calibration of a capacitive void fraction sensor for small diameter tubes, Int. J. Refrig., 36, 390-401, 2013.

Filippov, Y., Kakorin, I., and Kovrizhnykh, A.: New solutions to produce a cryogenic void fraction sensor of round cross-section and its applications, Cryogenics, 57, 55-62, 2013.

Hennes, J. and Barnard, L.: The Effects of Cryogenic Fuels on Capacitance Type Mass and Level Measurement, SAE Technical Paper 640222, Air Transport and Space Meeting, New York, 2730 April, 10 pp., 1964.

Jiang, Y. and Zhang, P.: Density determination of slush nitrogen by the improved capacitance-type densimeter, Exp. Therm. Fluid. Sci., 35, 328-337, 2011.

Kandlbinder, C., Fischerauer, A., Mösch, M., Helling, T., Fischerauer, G., and Siegl, M.: Original data of the publication: Capacitive gas-phase detection in liquid nitrogen, University of Bayreuth (Centre of Energy Technology - ZET), 2017.

Kügler, K.-J., Lindenberg, W., and Niehues, N.: Non-powerdissipating continuous level monitors for liquid nitrogen and helium, Cryogenics, 24, 63-66, 1984.

Matsumoto, K., Sobue, M., Asamoto, K., Nishimura, Y., Abe, S., and Numazawa, T.: Capacitive level meter for liquid hydrogen, Cryogenics, 51, 114-115, 2011.

Medeova, M., Pavlik, V., and Skyba, P.: Simple continuous level meter for cryogenic liquids, Cryogenics, 38, 289-291, 1998.

Mühlbacher-Karrer, S. and Zangl, H.: Light Weight Signal Processing for a Wireless Capacitive Sensing Platform for Mobile Applications, Proc. Sensor 2015, Nuremberg, Germany, 190-197, 2015.

Mukhopadhyay, M.: Fundamentals of cryogenic engineering, PHI Learning Pvt. Ltd., New Delhi, India, 2010.

Murphy, E. J. and Morgan, S. O.: The Dielectric Properties of Insulating Materials, Bell Systems Technical Journal, 14, 493-512, 1937.

Nurge, M. A., Youngquist, R., and Walters, D.: Capacitance based mass metering for cryogenic fluids, Cryogenics, 43, 501-506, 2003. 
Ohira, K.: Development of density and mass flow rate measurement technologies for slush hydrogen, Cryogenics, 44, 59-68, 2004.

Schlegl, T., Kröger, T., Gaschler, A., Khatib, O., and Zangl, H.: Virtual whiskers - Highly responsive robot collision avoidance, 2013 IEEE/RSJ Int'l. Conf. on Intelligent Robots and Systems, Tokyo, 5373-5379, 2013.

Siegl, M., Gerstmann, J., Schmidt, K., Becker, D., Lindner, G., Fischerauer, A., Kandlbinder, C., and Fischerauer, G.: Advanced Sensor technologies for cryogenic liquid propellant flow phenomena, Proc. 66th International Astronautical Congress (IAC), Jerusalem, 7 pp., 12-16 October, 2015.
Span, R., Lemmon, E., Jacobsen, R., Wagner, W., and Yokozeki, A.: A Reference Equation of State for the Thermodynamic Properties of Nitrogen for Temperatures from 63.151 to $1000 \mathrm{~K}$ and Pressures to $2200 \mathrm{MPa}$, J. Phys. Chem. Ref. Data, 29, 13611433, 2000.

Stolten, D. and Emonts, B.: Hydrogen Science and Engineering: Materials, Processes, Systems and Technology, Volume 1, WileyVCH, Weinheim, Germany, 2016.

Wang, F., Marashdeh, Q., Fan, L.-S., and Warsito, W.: Electrical Capacitance Volume Tomography: Design and Applications, Sensors 2010, 10, 1890-1917, 2010. 